\title{
THE RADIOCARBON CHRONOLOGY OF THE NEOLITHIC AND COPPER AGE OF NORTHERN ITALY
}

\begin{abstract}
Summary. Until twenty years ago the chronology of the Neolithic and Copper Age settlement of Northern Italy was almost exclusively based on the stratigraphical sequence of the Arene Candide cave in Liguria. The research carried out since the sixties has strongly increased our knowledge of the earliest farming communities and the first copper using people who inhabited the country between the end of the seventh and the beginning of the fourth millennium BP. This article considers the available evidence for this period which is now supported by a good set of radiocarbon dates.
\end{abstract}

\section{INTRODUCTION}

Our knowledge of the Neolithic and Copper Age settlement of Northern Italy has increased during the last twenty years largely due to the reexamination of the assemblages stored in national and local museums and to the excavation of some of the more important sites. Nevertheless, relatively little attention has been paid to the radiocarbon dating of the archaeological cultures of this region even though several articles have been devoted to this problem (Broglio 1972; Barfield 1974; Renfrew and Whitehouse 1974; Whitehouse 1978; Bagolini et al. 1979; Biagi 1980; Alessio et al. 1983; Sargent 1984; Bagolini and Biagi 1986; 1987a; Allegri et al. 1987).

THE CULTURAL/CHRONOLOGICAL SEQUENCE

The presence of Mesolithic hunter/gatherers in Northern Italy has been known since 1961 (Radmilli 1983) along the coast of the Trieste
Gulf (Cannarella and Cremonesi 1967). At the end of the sixties some sites were accidentally discovered in the Adige Valley around Trento. Since then programmed surveys have been carried out in the central eastern Alpine arch and along the northern Apennine watershed between Tuscany and Liguria, where dozens of Mesolithic camps have been discovered and partly excavated (Biagi et al. 1980; Bagolini et al. 1983; Baffico et al. 1983). The most complete series known so far are those of Grotta Benussi in the Trieste Karst (Cremonesi 1978-81) and Romagnano III in Trentino (Broglio 1971). At Grotta Benussi the appearance of industries with trapezes and notched blades of 'Castelnovian' type is dated to $7620 \pm 150$ BP (6600-6270 BC) (R-1044), and to $7850 \pm 60$ BP $(6781-6591 \quad \mathrm{BC})$ (R-1137) at Romagnano III (Figs. 1 and 2). Similar assemblages occur around the mid eighth millennium BP (R-1525a: $7380 \pm 130$ BP, 6400-6090 BC) in the Tuscan Apennines, 


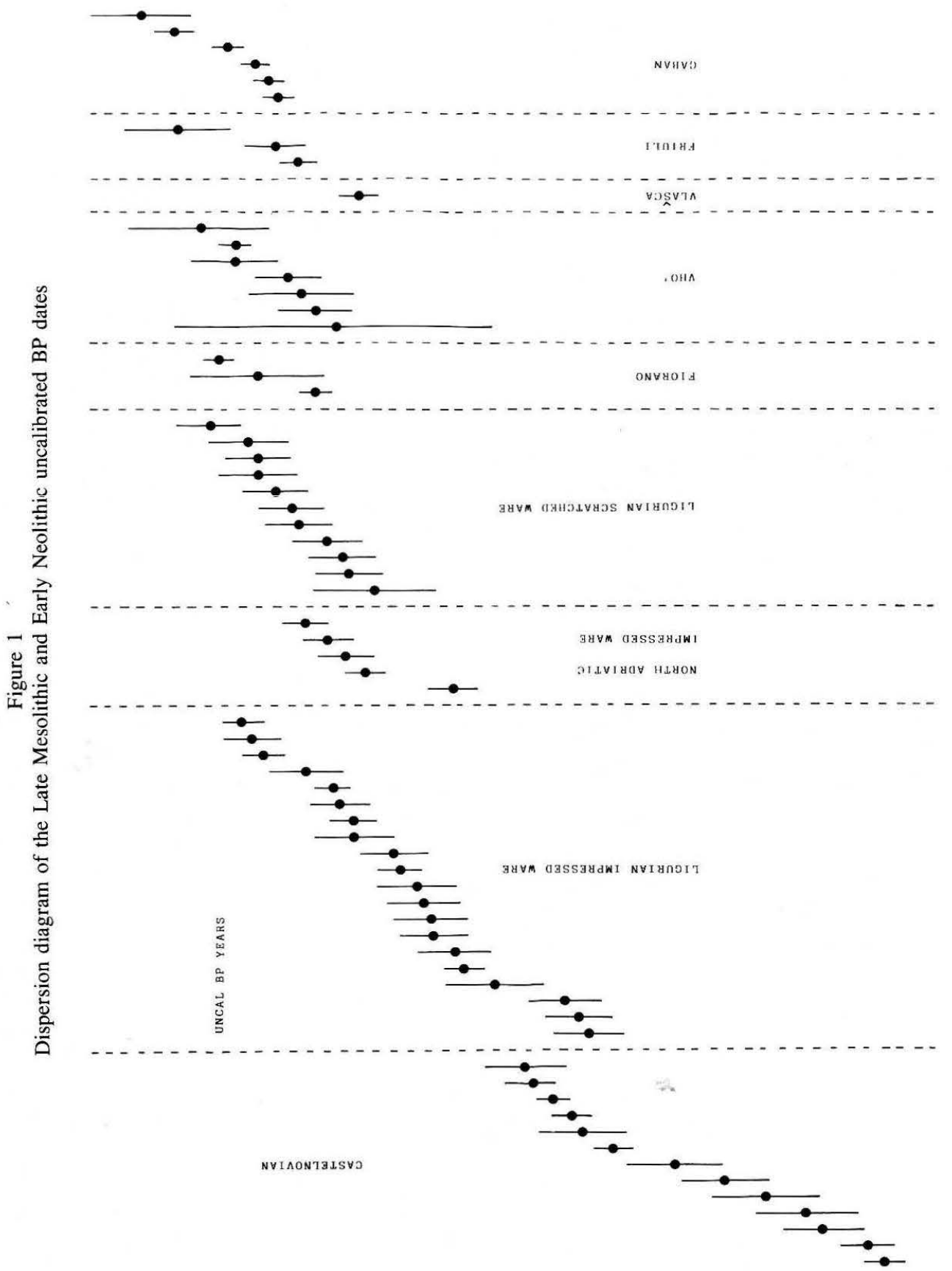

$99<\varepsilon-3 ! 5$ $\checkmark L L L \mathrm{~T}-\mathrm{U} I \mathrm{~B}$

T $8 \mathrm{~L}-\mathrm{H}$

$8 \angle L \mathrm{~T}-\mathrm{UT} \mathrm{T}$

$\angle L L I-U I \varepsilon$

$\forall 18 L-y$

Vsvst-u

VVVGt-r

ELEE-น।

vovor-y

$66 \angle \varepsilon \mathrm{L}-\mathrm{I}$

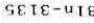

हटाG- IEH

द $ठ 2 Z-4$ व

86LE T-1

SDVI $1-1$

DODIT-I

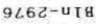

$89 \nabla-y$

०LEะ-u I

I 2 ¿-8

$8 \varepsilon[D-c]$

$8 G L-5 W$

อว. โ-วW

$6 \subseteq \angle-5 W$

IVIV-rT

ODID-rT

$0<0 z-80$

TOID-P]

6ย โิ

टटจट-8n

$869-y$

$\forall 869-y$

$66 \mathrm{~s}-\mathrm{y}$

ट LE $E-\mathrm{U}$ T

$\varepsilon \vee 9-4$

ช 9 I $\varepsilon-\mathrm{d}$

$\varepsilon 己 \varepsilon-\mathrm{z}$

9 I $\varepsilon-y$

ع. 9 -

โO $T-8$

$0 \varsigma \vee \varepsilon-u T g$

S $I \varepsilon-4$

S9z-

ชย $โ \varepsilon-y$

$\varepsilon$ เ $\varepsilon-\mathrm{t}$

$\angle 9 z-y$

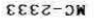

टยย己-วW

$\Delta \cap T \nabla-r T$

$\angle S L-D W$

$9 \angle Z \varepsilon-u I g$

$\checkmark ट \nabla z-80$

$\varepsilon \forall[\nabla-r]$

$9 S L-0 W$

$\varepsilon 己 \triangleright る-8 ก$

I $\angle 88$ LLZE-U T 8 $8 \nabla T \tau-\mathrm{y}$ 268-y

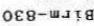
EจOI-y उDOI-y VSZS I$\forall \angle E \tau \mathrm{I}-\mathrm{y}$ $\triangle \nabla O I-Y$ $\angle 892$ I-I g $\angle E I T-y$ LEI I-Y 


\section{B. BAGOLINI AND P. BIAGI}

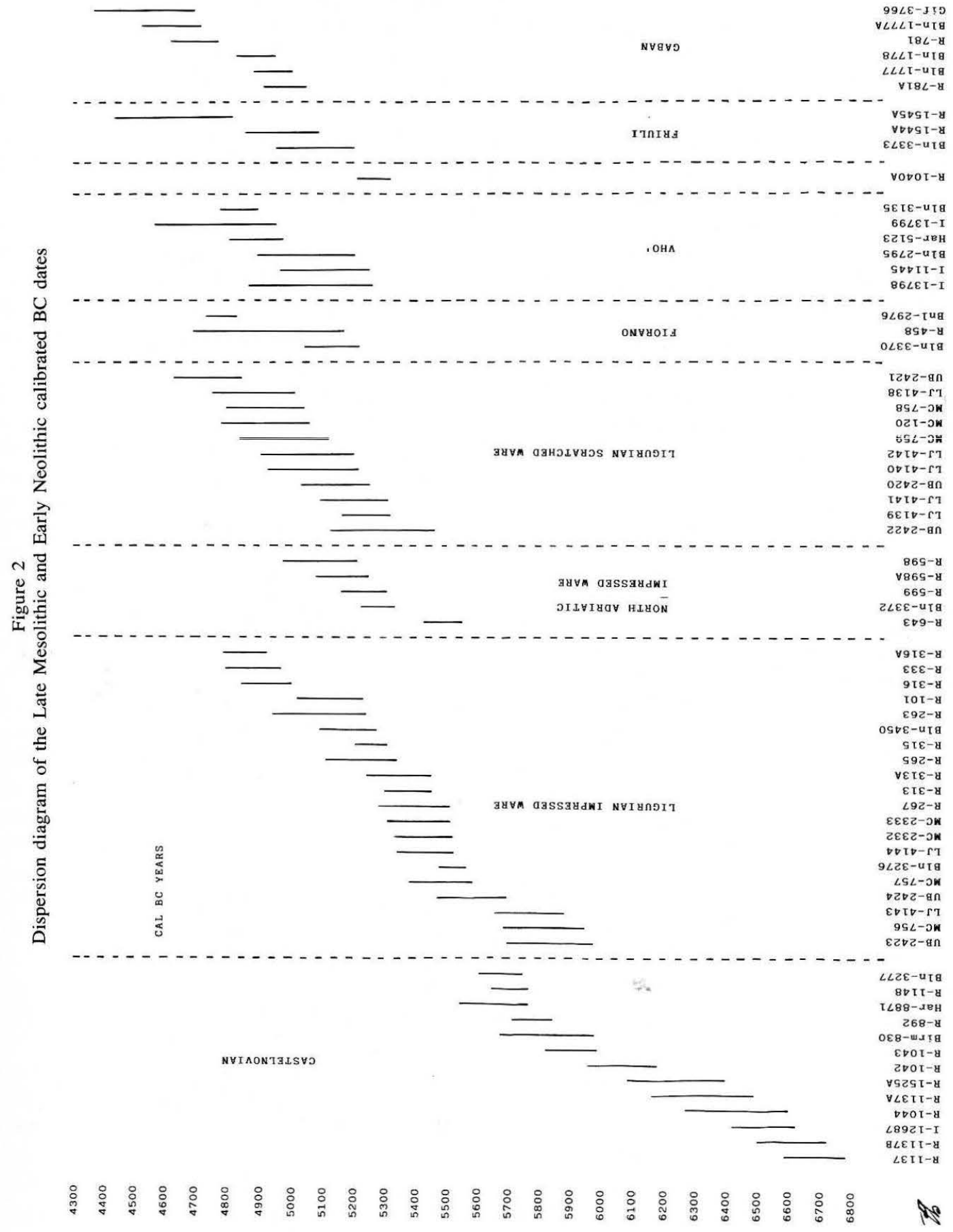


where the valley bottom site of Isola Santa was systematically resettled after the end of the Late Glacial. In the 'red earth' layer of Monte Bagioletto, a pass in the Emilian Apennines at $1700 \mathrm{~m}$ above sea level, the first trapezes make their appearance around $7670 \pm 120 \mathrm{BP}$ (6620-6420 BC) (I-12687) (Cremaschi et al. 1981-82). Mid and high altitude blade and trapeze 'Castelnovian' camps have also been excavated at Passo della Comunella (1619 m) in the Emilian Apennines (Birm-830: 6960土 130 BP, 5980-5680 BC), at Sopra Fienile Rossino in the central Pre-Alps (925 m) (Bln-3277: 6810 $\pm 70 \mathrm{BP}, 5749-5611 \mathrm{BC}$ ) (Accorsi et al. 1986) and at Laghetti del Crestoso, a hunting stand at $2000 \mathrm{~m}$ in the Brescian Alps (Har-8871: $6790 \pm 120$ BP, 5770-5550 BC) (Baroni et al. 1989). A layer with notched blades and trapezes was found in the Grottina dei Covoloni del Broion in the Berici Hills (Cattani 1977), quoted as R-892: $6930 \pm 60 \mathrm{BP}, 5850-5719 \mathrm{BC}$.

The origin and development of the Impressed Ware Culture along the western Ligurian (Bernabo' Brea 1956; Lewthwaite 1981; 1985; 1986; Biagi and Nisbet 1986) and the Northern Adriatic coastlines (Bagolini and von Eles 1978) has been discussed in several articles (Antoniazzi et al. 1986; Biagi 1987). The more ancient absolute dates so far known for this culture come from Arene Candide (UB-2423; $6980 \pm 115 \mathrm{BP}, 5980-5700 \mathrm{BC}$ ) and from the nearby Caverna Pollera (MC-756: $6950 \pm 100$ BP, 5950-5690 BC). More recent dates, covering almost the entire seventh millennium $\mathrm{BP}$, come from these as well as from four other western Ligurian caves of the Finalese and Val Pennavaira. Along the northwestern Adriatic coastline a set of $\mathrm{C} 14$ dates has been obtained from the Impressed Ware settlements of Maddalena di Muccia (Barker 1975), Ripabianca di Monterado (Broglio and Lollini 1963) and Fornace dei Cappuccini (Antoniazzi et al. 1986). Maddalena di Muccia gave the result of $6580 \pm 75 \mathrm{BP}(5562-5434 \mathrm{BC})$ (R-643), while the other two sites fall into the second half of the seventh millennium BP. Similar dates, ranging between the last centuries of the seventh and the beginning of the sixth millennia BP were obtained from sites of the various Groups of the Po and Friuli plains and Trentino/South Tyrol, namely Fiorano, Vhò, Fagnigola and Gaban. As revealed by layer $8 b$ of Grotta dei Ciclami (R-1040a: 6300 $\pm 60 \mathrm{BP}, 5328-5225 \mathrm{BC}$ ), the Vlašca or Vasi a Coppa Group of the Trieste Karst is also to be attributed to the same horizon.

The end of the Impressed Ware Culture in Liguria is marked by the appearance of a new pottery style of black burnished fabric, with scratched ornamentations, similar to the ceramics of the Po Plain Groups of the end of the seventh millennium BP (Maggi 1977). The start of the sixth millennium BP sees the spread of the Square Mouth Pottery Culture which flourished over the whole of Northern Italy a few centuries later. This culture was first subdivided into three main phases (Barfield 1973) and later into pottery styles based on the ceramic decorations (Bagolini et al. 1979). Several C14 dates are now available for this culture. Many of them come from the lake-side village of Fimon Molino Casarotto in the Berici Hills (Bagolini et al. 1973; Barfield and Broglio 1986) and others have been obtained from the Arene Candide and Romagnano III stratigraphical sequences. Dalmatian-type ornamentations always occur around the middle of the sixth millennium BP. This happens at many sites in the Po Valley and the Alpine arch, including Campegine, La Vela (Bagolini and Biagi 1976), Romagnano and Castelgrande in the Ticino Canton $(\mathrm{CH})$ (Donati 1986). A few C14 dates are also available for the Isolino facies which, according to the material assemblage, is more or less contemporary with the spiral/meander 
style of the SMP Culture. In the Trieste Karst, a region in the sphere of the Dalmatian and Danubian Cultures, layer 8 of Grotta del Mitreo, with Danilo type pottery, has been dated to $5770 \pm 60 \mathrm{BP}(4737-4550 \mathrm{BC})$ (R-904) and to $5770 \pm 50 \mathrm{BP}(4729-4574 \mathrm{BC})$ (R-904a) (Figs. 3 and 4). A few C14 dates are also available for the last moments of the SMP culture as it is known from eastern Lombardy, the Veneto and Trentino regions. The advent of the Chassey and the slightly later Lagozza Cultures (Biagi 1982; Bagolini 1986) happened around the end of the sixth and the beginning of the fifth millennia BP. At present we have only two dates for the pure Chassey Culture (Figs. 5 and 6). The first comes from Arene Candide (R-104: $5075 \pm 45$ BP, 3968-3805 $\mathrm{BC})$ and the second from the open settlement of Spilamberto (Bagolini 1981) along the northern fringe of the Emilian Apennines (I-11817: $4995 \pm 100 \mathrm{BP}, 3950-3690 \mathrm{BC}$ ). A more extensive set is known for Lagozza, where the eponymous site produced the best evidence (Barfield 1974).

The number of $\mathrm{C} 14$ dates known for the Chalcolithic has greatly increased during the last decade. The heterogeneity of the site types and locations as well as the material employed for dating is clearly visible in Table 1 'Chalcolithic'. Human bones have been used from the burial cave of Val Frascarese (Maggi and Formicola 1978). Of extreme interest are the results from the wooden pick from the prehistoric Ligurian mine of Libiola (Isetti 1964) discovered at the end of the last century (Issel 1892) which confirm that it had already been exploited during the Chalcolithic (Bln3367: $4610 \pm 50$ BP, 3382-3342 BC; Gif-7213: $4490 \pm 90$ BP, 3354-3035 BC) (Maggi and Vignolo 1989). Four Beaker sites produced only acceptable $\mathrm{C} 14$ dates, while those from Arma di Nasino in Liguria (Barfield 1974) and from the megalithic cemetery of Aosta (Mezzena 1981) are highly questionable.

\section{CONCLUSIONS}

The beginning of the Atlantic period is marked by the appearance of Mesolithic communities with a Castelnovian tool-kit characterised by trapezes, notched blades and other instruments chipped from pyramidal bladelet cores. The C14 chronology of these communities is well known, even though a gap still exists around the final stages in the development of this culture. The more recent dates belong to the beginning of the seventh millennium BP, while the first pottery-making peoples settled in the Po Valley around the end of the same millennium. A different picture is apparent for Liguria, where the Impressed Ware Culture makes its appearance at the beginning of the seventh millennium BP as documented both from the Arene Candide and Pollera caves. Recent discoveries along the nearby French coastline demonstrate that most of the Late Mesolithic and Early Neolithic sites were destroyed by the last sea-level rise (Geddes et al. 1983; Mills 1983). Many Castelnovian camps are known at middle/high altitudes on the Alpine (Baroni et al. 1989) and Apennine Ligurian watershed (Biagi et al. 1989), while only cave sites of the Impressed Ware Culture exist in this latter region. The radiocarbon evidence suggests a partial contemporaneity of these cultural traditions in Liguria, and is supported by contemporary ethnographic data (Orme 1981). More recent dates come from the settlements of the Adriatic stream of the Impressed Ware Culture and by the Vlašca cave of Grotta dei Ciclami. Imports of Figulina painted flasks of Ripoli style are recorded from the late seventh millennium $\mathrm{BP}$ layer 13 of the Arene Candide sequence (Tinè 1974), during the centuries when the central Italian Ripoli and the north Italian Fiorano Cultures seem to have reached their apex. Relationships were maintained between the Po Plain SMP Culture and that of the peninsular 


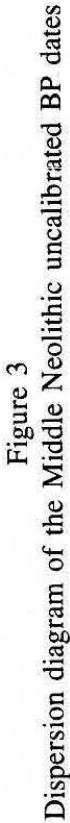

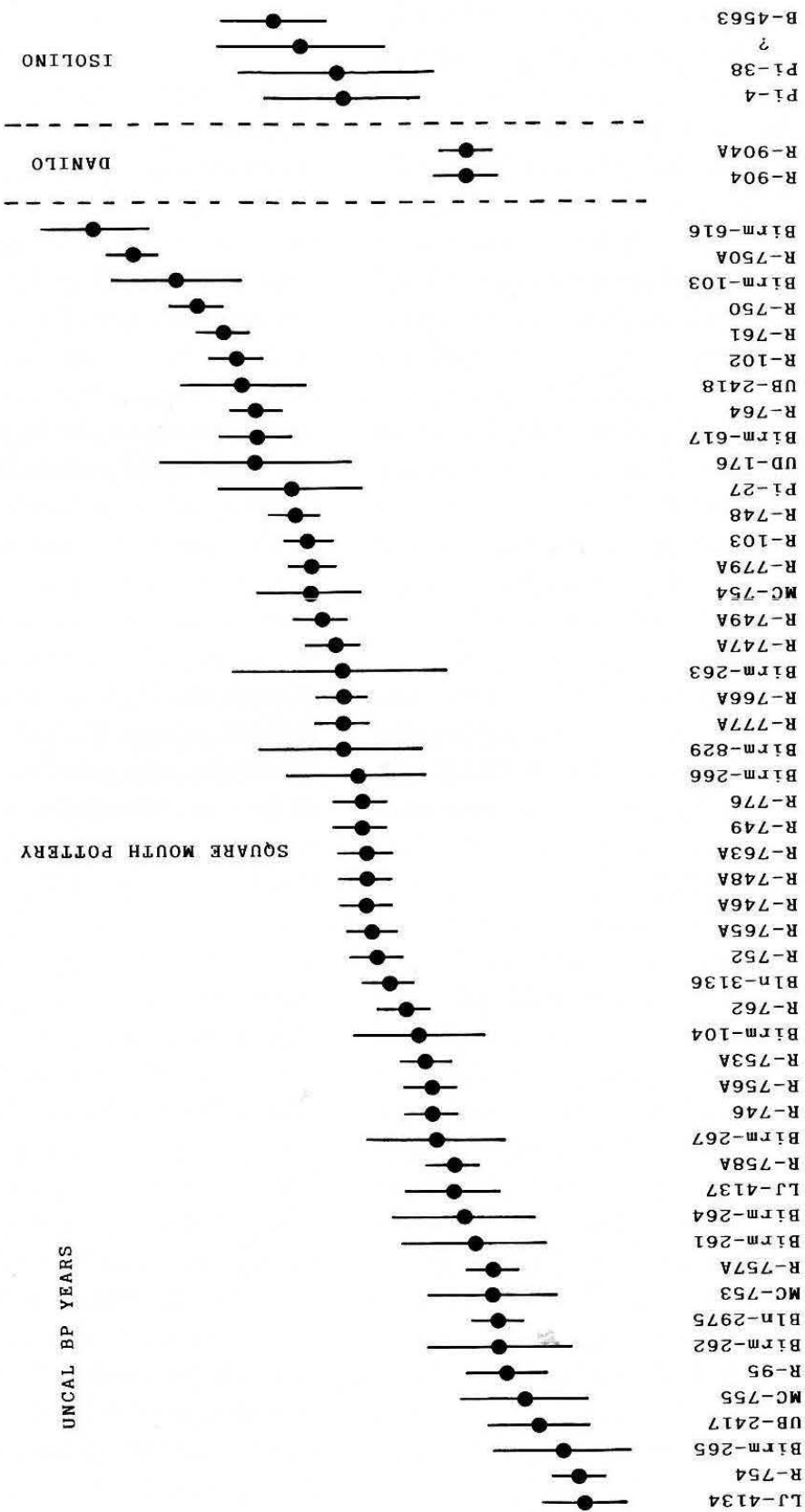

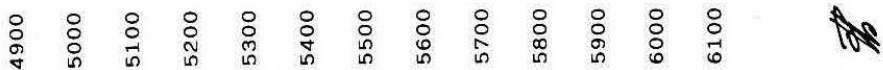




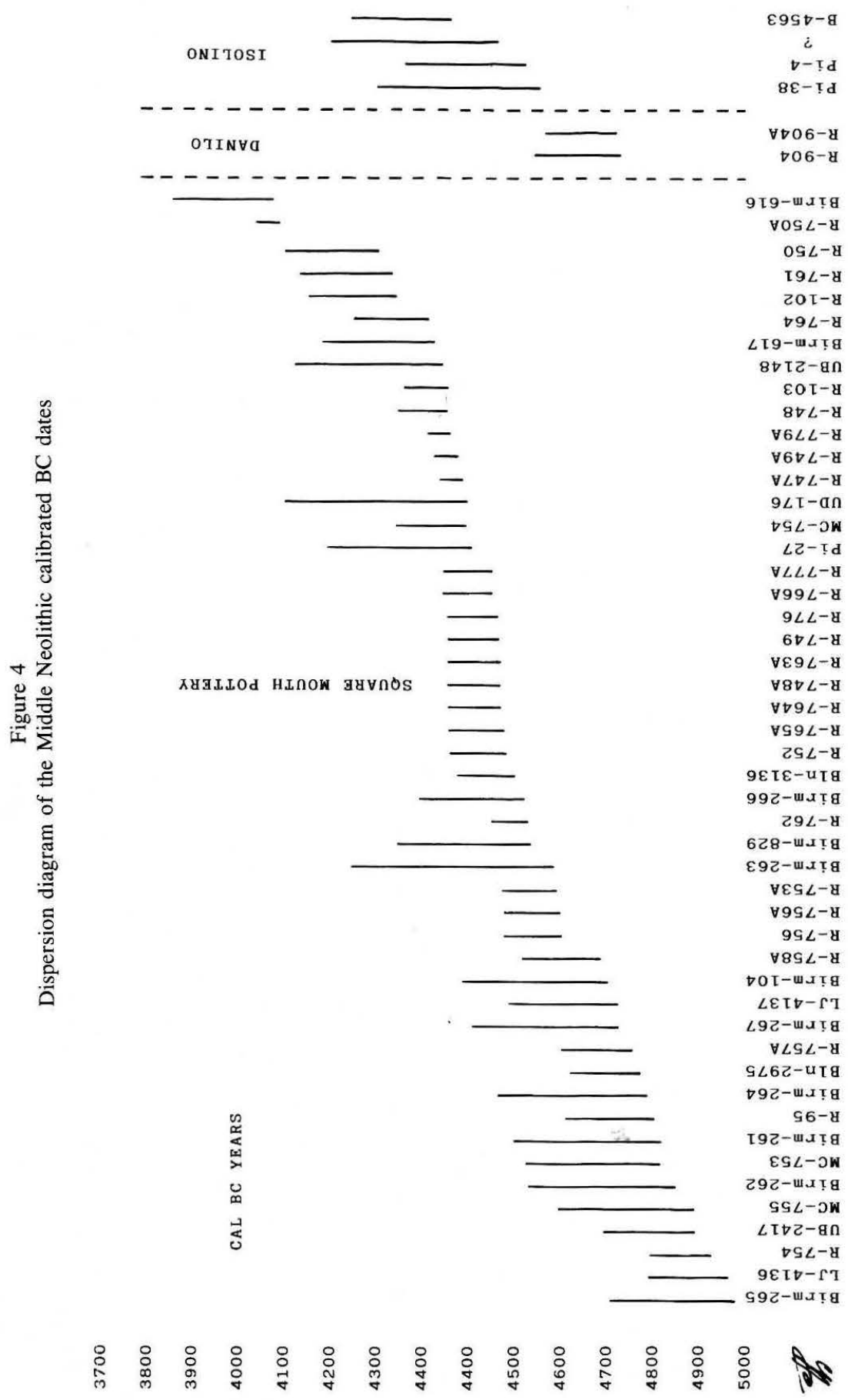




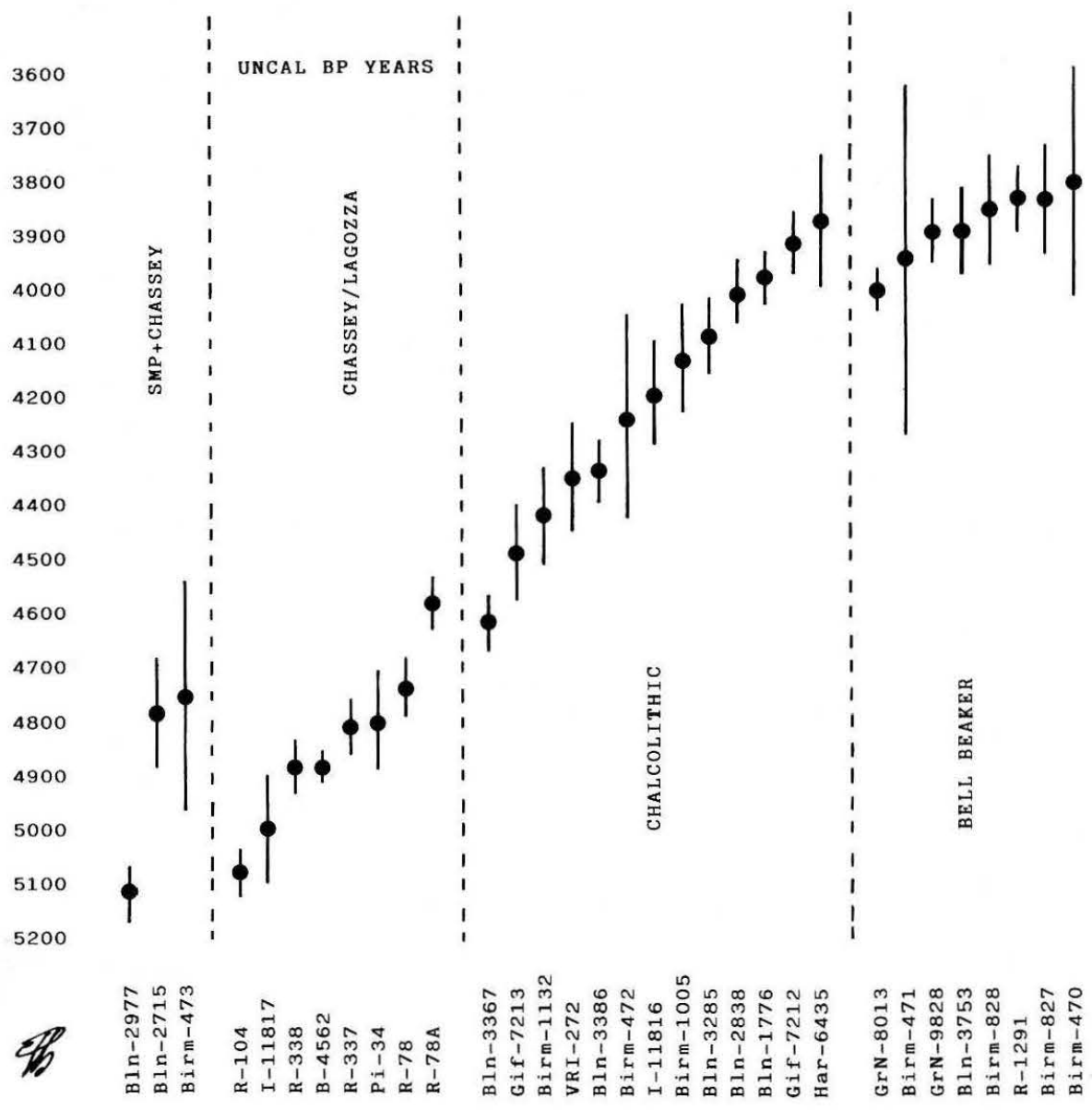

Figure 5

Dispersion diagram of the Late Neolithic and Copper Age uncalibrated BP dates

Serra d'Alto during the following millennium (Whitehouse 1978; 1985). Serra d'Alto unpainted and painted pottery has been recorded from typical Square Mouth sites, also in the shape of complete vessels at La Vela, for instance (Bagolini 1989). The role played by the Adige valley in the transalpine trade has been demonstrated by the discovery of central European artefacts at La Vela and Rivoli (Barfield 1970) and by characteristic orna- mentation on some square mouth vases of South Tyrol (Niederwanger 1984).

The radiocarbon evidence for the mid sixth/ mid fifth millennium BP is still rather scanty. In fact it is impossible to follow the advent and development of Chassey and Lagozza Cultures and the fall of the SMP on the basis of the data currently available. The spread of the Diana Culture further to the north, as far as Romagna, also occurred in this period. 


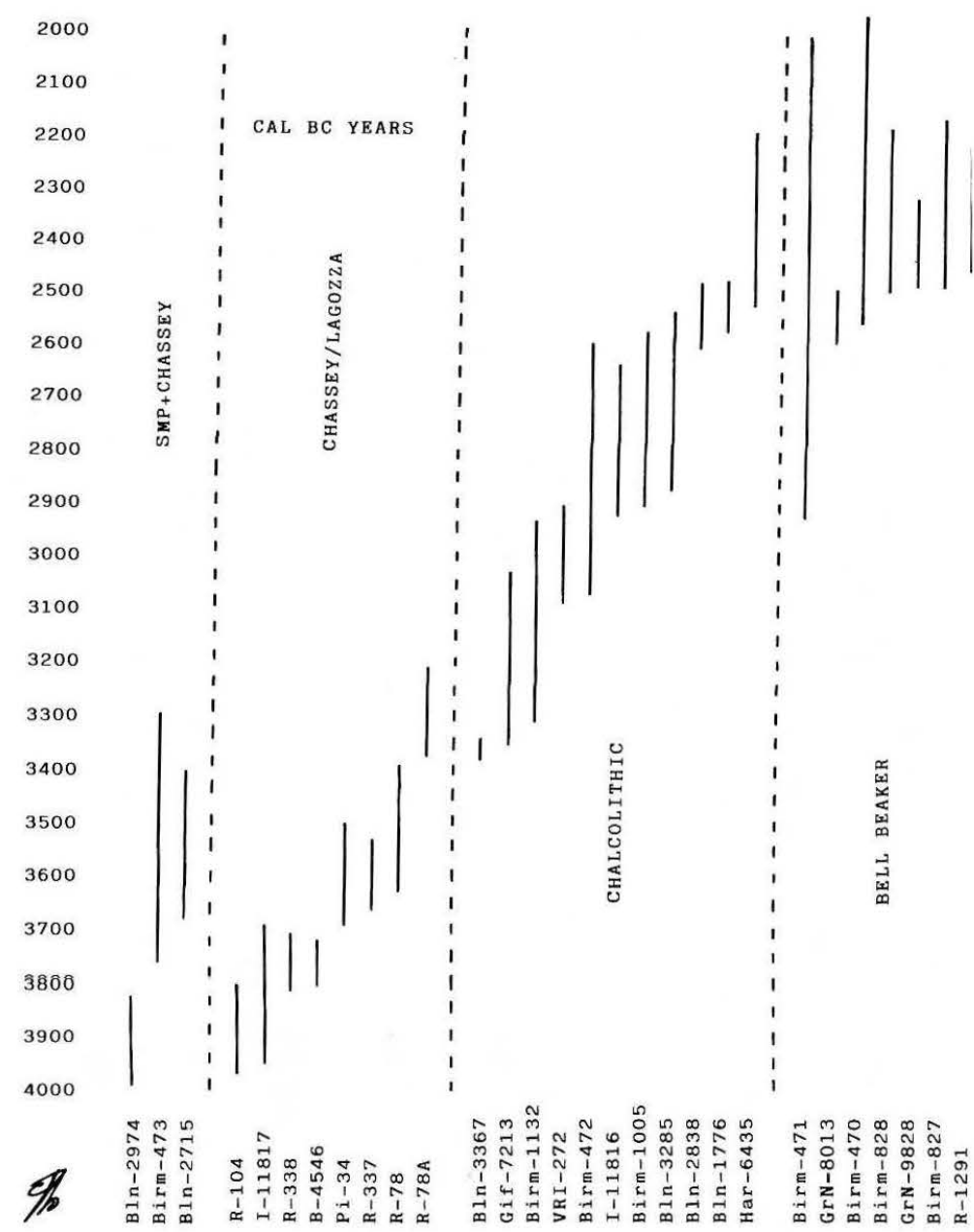

Figure 6

Dispersion diagram of the Late Neolithic and Copper Age calibrated BC dates

The Copper Age of northern Italy is represented by the cemeteries of Remedello type (Colini 1898-1902), by the settlements and graveyards of the Spilamberto facies distributed south of the river Po (Bagolini 1981) and by the burial caves and rock shelters of the alpine and apennine regions (Barfield 1985; Cocchi Genick and Grifoni Cremonesi 1985). The C14 dates now available indicate that between the second half of the fifth and the beginning of the fourth millennia BP, several facies, strongly related to each other, were present in northern Italy. Comparisons can be extended to the Rinaldone and Gaudo Cultures of the Tyrrhenian coast which produced bifacial, tanged, flint arrowheads, copper halberds (Barfield 1969) and knives very similar to those from Remedello. Knives 
TABLE 1

LIST OF THE RADIOCARBON DATES

Site name

CASTELNOVIAN LATE MESOLITHIC
Unca1 BP years Ca1 BC years
$(1 \boldsymbol{\sigma})$
Lab $n$

$7850 \pm 60$
$7500 \pm 160$
$7800 \pm 80$
$7050 \pm 60$
$7230 \pm 140$
$7620 \pm 150$
$7670 \pm 120$
$7380 \pm 130$
$6960 \pm 130$
$6970 \pm 60$
$6870 \pm 50$
$6810 \pm 70$
$6790 \pm 120$

LIGURIAN IMPRESSED WARE CULTURE

$$
\begin{aligned}
& 6980 \pm 115 \\
& 6910 \pm 110 \\
& 6700 \pm 145 \\
& 6520 \pm 100 \\
& 6220 \pm 55 \\
& 6950 \pm 100 \\
& 6580 \pm 110 \\
& 6610 \pm 60 \\
& 6470 \pm 120 \\
& 6420 \pm 65 \\
& 6400 \pm 105 \\
& 6280 \pm 120 \\
& 6280 \pm 70 \\
& 6140 \pm 110 \\
& 6015 \pm 65 \\
& 5980 \pm 85 \\
& 5955 \pm 65 \\
& 6240 \pm 90 \\
& 6510 \pm 110 \\
& 6490 \pm 110
\end{aligned}
$$

$5980-5700$

$5890-5660$

$5700-5480$

5530-5350

$5241-5163$

5950-5690

$5590-5390$

5575-5480

5520-5290

5461-5311

5460-5250

5350-5120

5317-5217

5250-4950

5010-4847

4979-4797

4930-4787

5283-5098

5530-5340

5520-5320

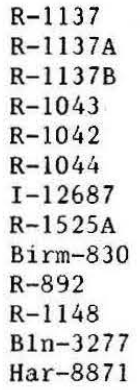

UB-2423

LJ-4143

UB-2424

LJ-4144

R-101

MC -756

MC-757

B 1n-3276

R-267

R-313

$\mathrm{R}-313 \mathrm{~A}$

R-265

R-315

$\mathrm{R}-263$

$\mathrm{R}-316$

$\mathrm{R}-333$

$\mathrm{R}-316 \mathrm{~A}$

B 1 n-3450

MC -2332

$\mathrm{MC}-2333$

NORTH ADRIATIC IMPRESSED WARE CULTURE

Maddalena di Muccia
Cappuccini
Ripabianca
Ripabianca
Ripabianca

LIGURIAN "SCRATCHED" WARES

Arene Candide

Arene Candide

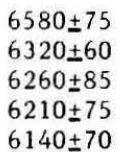

$6345 \pm 180$ $6270 \pm 100$
5562-5434

$5339-5230$

5312-5167

5256-5085

5223-4978

5470-5130

5330-5170
$R-643$

B1n-3372

R-599

R-598A

R-598 
Charcoa1

Charcoal

Charcoa 1

Charcoal

Charcoa1

Charcoal

Charcoal

Charcoal

Charcoal

Charcoal

Charcoal

Charcoal

Charcoal

Charcoal

Charcoal

Charcoal

Charcoa1

Charcoal

Charcoa 1

Charcoa1

Charcoal

Charcoal

Charcoal

Charcoa 1

Charcoal

Charcoal

Charcoa 1

Charcoal

Charcoal

Charcoal

Charcoal

Charcoal

Charcoal

Charcoal

Charcoal

Charcoal

Charcoal

Charcoal

Charcoal

Open
Open
Open
Open
Open

Cave
Cave

Pit

Pit

Pit

Pit

Pit

Layer 14

Layer 14-13

Layer 14

Layer 15-14

Layer 26-25

Layer XXX

Layer XXIX

Layer 2

Layer IXi

Layer VIII

Layer VIII

Layer IXs

Layer IXs

Layer VIIIa

Layer IXm

Layer $X$

Layer IXm

Below layer $\mathrm{V}$

Hearth

Hearth

\author{
Alessio et a1. 1978: 249 \\ Alessio et a1. 1978: 249 \\ Alessio et a1. 1978: 249 \\ Alessio et a1. 1978: 251 \\ Alessio et a1. 1978: 251 \\ Alessio et a1. 1978: 251 \\ Cremaschi et al. 1981-82: 18 \\ Alessio et a1. 1978: 251 \\ Biagi et al. 1980: 17 \\ Alessio et a1. 1978: 251 \\ Alessio et a1. 1978: 248 \\ Accorsi et a1. 1986: 251 \\ Baroni et al. 1989 (in press)
}

Biagi and Maggi 1983: 166

Maggi 1977: 211

Mallory pers. comm. 1979

Maggi 1977: 211

Alessio et a1. 1966: 402

Tinè 1974: 52

Tinè 1974: 52

Biagi et al. 1987: 11

Alessio et al. 1968: 354

Alessio et al. 1968: 355

Alessio et al. 1968: 355

Alessio et al. 1968: 354

Alessio et a1. 1968: 355

Alessio et a1. 1968: 354

Alessio et a1. 1968: 355

Alessio et a1. 1968: 353

Alessio et a1. 1968: 355

Girod 1989 (in press)

Odetti 1984: 112

odetti 1984: 112

Alessio et al. 1970: 603

Bagolini and Biagi 1987b: 217

Alessio et a1. 1970: 602

Alessio et a1. 1970: 602

Alessio et al. 1970: 602 
Site name

Arene Candide Arene Candide Arene Candide Arene Candide Arene Candide Arene Candide Arene Candide Pollera

Pollera

VLAŠCA GROUP

Ciclami

$6300 \pm 60$

$6250 \pm 100$

$6205 \pm 105$

$6120 \pm 100$

$6100 \pm 100$

$6000 \pm 120$

$5970 \pm 120$

$5855 \pm 95$

$6050 \pm 100$

$6000 \pm 100$

FRIULI EARLY NEOLITHIC GROUPS
Sammardenchia

Fagnigola

Fagnigola

FIORANO CULTURE

Lugo di Romagna
Chiozza
Savignano

VHO' GROUP

Vhò

Vhò

Vhò

Travo

Travo

Ostiano Dugali Alti

Cecima

GABAN GROUP

\section{Riparo Gaban}

Riparo Gaban

Riparo Gaban

Riparo Gaban

Romagnano III

Romagnano III

SQUARE MOUTH POTTERY CULTURE

$6120 \pm 60$

$6050 \pm 90$

$5760 \pm 160$

$6170 \pm 50$

$6000 \pm 200$

$5880 \pm 50$

$6235 \pm 470$

$6170 \pm 110$

$5930 \pm 50$

$6130 \pm 160$

$5830 \pm 210$

$6090 \pm 100$

$5930 \pm 130$

$6030 \pm 45$

$5990 \pm 45$

$5750 \pm 60$

$5650 \pm 150$

$6060 \pm 50$

$5810 \pm 50$

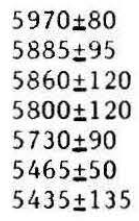

$5970 \pm 80$

$5885 \pm 95$

Arene Candide

Arene Candide

Arene Candide

Arene Candide

Arene Candide

Arene Candide
Cal BC years

(1)

$5320-5100$
$5260-5040$
$5230-4930$
$5210-4910$
$5070-4780$
$5020-4750$
$4850-4627$
$5130-4840$
$5050-4800$

LJ-4141

UB-2420

LJ -4140

$\mathrm{LJ}-4142$

MC -120

LJ -4138

UBB-2421

$\mathrm{MC}-759$

MC -758

5370-5154

$R-1040 A$

5211-4958

5097-4857

4820-4440

B 1 n-3373

$\mathrm{R}-1544 \mathrm{~A}$

$\mathrm{R}-1545 \mathrm{~A}$

5224-5062

5180-4690

4831-4730

B 1 n -3370

$\mathrm{R}-458$

B1n-2976

5600-4640

$5260-4970$

4900-4781

$5270-4870$

4960-4470

$5210-4900$

4980-4710

I-11444

I -11445

B1n-3135

I-13798

I-13799

B1n-2795

Har -5123

5012-4883

4954-4829

4721-4527

4700-4370

5061-4918

4774-4618

B1n-1777

B1n-1778

B $1 n-1777 \mathrm{~A}$

Gif-3766

$\mathrm{R}-781 \mathrm{~A}$

$\mathrm{R}-781$

LJ -4136

UB-2471

MC -755

$\mathrm{MC}-753$

LJ-4137

R-103

Pi-27 


$\begin{array}{lll}\text { Material } & \text { Site type } & \text { Feature } \\ & & \\ & & \\ \text { Charcoal } & \text { Cave } & \text { Layer 13 } \\ \text { Charcoal } & \text { Cave } & \text { Layer 13A } \\ \text { Charcoal } & \text { Cave } & \text { Layer 13 } \\ \text { Charcoal } & \text { Cave } & \text { Layer 13 } \\ \text { Charcoal } & \text { Cave } & \text { Layer 13 } \\ \text { Charcoal } & \text { Cave } & \text { Layer 13 } \\ \text { Charcoal } & \text { Cave } & \text { Layer 13B } \\ \text { Charcoal } & \text { Cave } & \text { Layer XXIII } \\ \text { Charcoal } & \text { Cave } & \text { Layer XXIV }\end{array}$

Charcoal

Charcoal

Charcoal

Charcoal

Charcoal Charcoal Charcoal

\begin{abstract}
Charcoal
Charcoal

Charcoal

Charcoal

Charcoal

Charcoa1

Charcoal
\end{abstract}

Charcoal

Charcoal

Charcoa 1

Charcoal

Charcoal

Charcoal

Charcoal

Charcoa1

Charcoal

Charcoal

Charcoal

Charcoal

Charcoal

$\begin{array}{ll}\text { Open site } & \text { Pit XVIII } \\ \text { Open site } & \text { Pit XVIII } \\ \text { Open site } & \text { Pit XXXII } \\ \text { Open site } & \text { Pit } \\ \text { Open site } & \text { Pit } \\ \text { Open site } & \text { Pit III } \\ \text { Open site } & \text { Pit }\end{array}$

Rock-she1ter Layer D2 Rock-shelter Layer D8 Rock-she1ter Layer D2 Rock-shelter Layer D8 Rock-shelter Layer T4 Rock-shelter Layer T4

$\begin{array}{ll}\text { Cave } & \text { Layer } 12 \\ \text { Cave } & \text { Layer } 12 \\ \text { Cave } & \text { Layer } 12 \mathrm{c} \\ \text { Cave } & \text { Layer } 12 \mathrm{a} \\ \text { Cave } & \text { Layer } 12 \\ \text { Cave } & \text { Layer } 19-16 \\ \text { Cave } & \text { Layer } 20\end{array}$

Reference

Maggi 1977: 211

Mallory pers. comm. 1979

Maggi 1977: 211

Maggi 1977: 211

Tinè 1974: 52

Maggi 1977: 211

Mallory pers. comm. 1979

Tinè 1974: 52

Tinè 1974: 52

Alessio et al. 1978: 86

Bagolini et a1. 1987: 412

Biagi 1985: 19

Biagi 1985: 19

Bagolini and Biagi 1987b: 219

Alessio et a1. 1970: 601

Biagi and Nisbet 1987: 14

Biagi 1979: 35

Biagi 1979: 35

Biagi 1987b: 388

Bernabò Brea 1986: 173

Bernabò Brea 1986: 173

Biagi 1987: 474

Simone 1987: 474

Bagolini and Biagi 1987: 427

Bagolini and Biagi 1987: 427

Bagolini and Biagi 1987: 427

Delibrias pers. comm. 1976

Alessio et a1. 1978: 81

Alessio et a1. 1978: 81

Maggi 1977: 211

Mallory pers. comm. 1979

Tinè 1974: 52

Tinè 1974: 52

Maggi 1977: 211

Alessio et a1. 1966: 402

Alessio et a1. 1966: 402 
Site name

Arene Candide

Arene Candide

Arene Candide

Aisone

Casalmoro

Travo

Campegine

Fimon M.C.

Fimon M.C.

Fimon M.C.

Fimon M.C.

Fimon M.C.

Fimon M.C.

Fimon M.C.

Fimon M.C.

Fimon M.C.

Fimon M.C.

Fimon M.C.

Fimon M.C.

Fimon M.C.

Fimon M.C.

Fimon M.C.

Fimon M.C.

Fimon M.C.

Fimon M.C.

Fimon M.C.

Fimon M.C.

Fimon M.C.

Fimon M.C.

Fimon M.C.

Fimon M.C.

Fimon M.C.

Fimon M.C.

Fimon M.C.

Fimon M.C.

Rivoli

Rivoli

Rivoli

Rivoli

Romagnano II I

Romagnano III

Romagnano III

La Vela

"ISOLINO" FACIES

Isolino

Isolino

Isolino

Castelgrande ( $\mathrm{CH}$ )

LATE SMP WITH CHASSEY/LAGOZZA
Uncal BP years

$5345 \pm 120$

$5335 \pm 50$

$5470 \pm 100$

$5825 \pm 75$

$5810 \pm 50$

$5610 \pm 50$

$5530 \pm 150$

$5930 \pm 130$

$5820 \pm 135$

$5800 \pm 50$

$5780 \pm 135$

$5750 \pm 135$

$5730 \pm 50$

$5700 \pm 130$

$5690 \pm 50$

$5690 \pm 50$

$5640 \pm 50$

$5570 \pm 50$

$5570 \pm 50$

$5570 \pm 50$

$5560 \pm 50$

$5555 \pm 130$

$5525 \pm 200$

$5510 \pm 50$

$5490 \pm 50$

$5440 \pm 50$

$5310 \pm 50$

$5260 \pm 50$

$5140 \pm 50$

$5530 \pm 50$

$5580 \pm 50$

$5370 \pm 50$

$5680 \pm 50$

$5960 \pm 50$

$5590 \pm 50$

$5665 \pm 125$

$5370 \pm 70$

$5220 \pm 120$

$5070 \pm 100$

$5560 \pm 50$

$5530 \pm 50$

$5470 \pm 50$

$5370 \pm 180$

$5534 \pm 144$

$5526 \pm 180$

$5460 \pm 155$

$5410 \pm 100$
Cal BC years

(1)

4350-4030

4250-4058

4400-4250

4805-4617

4774-4618

4506-4381

4540-4250

4980-4710

4850-4530

4762-4604

4820-4500

4790-4470

4689-4518

4730-4410

4603-4482

4603-4482

4535-4456

4475-4362

4475-4362

4475-4362

$4470-4359$

4530-4300

4590-4150

4387-4341

4371-4334

4354-4249

4242-4040

4212-4006

4000-3951

4455-4349

4481-4365

4327-4159

4594-4477

4923-4797

4487-4368

4700-4390

4332-4087

3990-3770

4470-4359

4455-4349

4364-4320

4400-4010

$4530-4270$

4560-4210

4470-4110

$4370-4150$
Lab $n$

UB-2418

R-102

MC -754

R-95

B1n-2975

B1n-3136

Birm-829

Birm-265

Birm-262

$\mathrm{R}-757 \mathrm{~A}$

Birm-261

Birm-264

R-758A

Birm-267

R-746

R-756A

R-762

$\mathrm{R}-764 \mathrm{~A}$

$\mathrm{R}-748 \mathrm{~A}$

R-763A

R-749

Birm-266

Birm-263

$\mathrm{R}-747 \mathrm{~A}$

$\mathrm{R}-749 \mathrm{~A}$

$\mathrm{R}-748$

$\mathrm{R}-761$

$\mathrm{R}-750$

$\mathrm{R}-750 \mathrm{~A}$

$\mathrm{R}-766 \mathrm{~A}$

$\mathrm{R}-765 \mathrm{~A}$

$\mathrm{R}-764$

$\mathrm{R}-753 \mathrm{~A}$

R-754

R-752

Birm-617

Birm-617

Birm-103

Birm-616

R-776

$\mathrm{R}-777 \mathrm{~A}$

$\mathrm{R}-779 \mathrm{~A}$

UD-176

$$
\begin{gathered}
\mathrm{Pi}-4 \\
\mathrm{Pi}-38 \\
?
\end{gathered}
$$$$
\text { B- } 4563
$$ 
Feature

Layer 12

tharcoal

Charcoal

Charcoal

Charcoal

Charcoal

Charcoal

Charcoal

Charcoa1

Charcoal

Charcol

Charcoal

Charcoal

Charcoal

Charcoal

Charcoal

Charcoal

Charcoal

Charcoal

Charcoa1

Wood

Charcoal

Charcoal

Charcoal

Charcoal

Charcoal

Charcoal

Charcoal

Charcoal

Charcoal

Charcoal

Charcoa 1

Wood

Wood

Charcoal

Wood

Charcoal

Charcoal

Bone

Charcoal

Charcoal

Charcoal

Charcoal

Charcoal

Wood

Wood

Wooder tool

Charcoal

\begin{abstract}
Cave
Cave

Cave

Cave

Open site

Open site

Open site

Lake dwe1ling

Lake dwelling

Lake dwelling

Lake dwe 11 ing

Lake dwe11ing

Lake dwe11ing

Lake dwelling

Lake dwelling

lake dwe 11 ing

Lake dwelling

Lake dwelling

Lake dwelling

Lake dwe1ling

Lake dwel1ing

Lake dwe11ing

Lake dwe11ing

Lake dwe11ing

Lake dwelling

lake dwe11ing

Lake dwelling

Lake dwe11ing

Lake dwe11ing

Lake dwe 11 ing

Lake dwe 11 ing

lake dwelling

Lake dwelling

Lake dwe11ing

lake dwelling

Open site

Open site

Open site

Open site

Rock-she1ter

Rock-she1ter

Rock-she1ter

Open site
\end{abstract}

Lake dwe11ing

Lake dwe11ing

Lake dwe1ling

Open site
House 1
Layer 24-21

Layer 8

Pit 1

Pit

Pit

House 1

House 1

House 1

House 1

House 1

House 1

House 1

House 1

House 1

House 1

House 1

House 1

House 1

House 1

House 1

House 1

House 1

House 1

House 1

House 1

House 1

House 2

House 2

House 2

House 3

Site 6

Site 1

Pit Z

Pit PQR

Pit L

Pit PQR

Layer T1

Layer $\mathrm{T} 1$

Layer T2

Layer 1

Structure

Structure

cm 260 depth

Layer 13
Reference

Mallory pers. comm. 1979

Alessio et a1. 1966: 402

Tinè 1974: 52

Alessio et al. 1965: 213

Quitta pers. comm. 1984

Bernabò Brea 1987: 173

Barfield pers. comm. 1978

Shotton and Williams 1973: 11

Shotton and Williams 1973: 11

Alessio et a1. 1974: 359

Shotton and williams 1973: 11

Shotton and Williams 1973: 11

Alessio et a1. 1974: 360

Shotton and Williams 1973: 11

Alessio et a1. 1974: 359

Alessio et a1. 1974: 359

Alessio et a1. 1974: 360

Alessio et a1. 1974: 359

Alessio et a1. 1974: 359

Alessio et a1. 1974: 360

Alessio et a1. 1974: 360

Shotton and Williams 1973: 11

Shotton and Williams 1973: 11

Alessio et a1. 1974: 359

A1essio et a1. 1974: 360

Alessio et a1. 1974: 359

Alessio et al. 1974: 360

Alessio et al. 1974: 360

Alessio et al. 1974: 360

Alessio et a1. 1974: 361

Alessio et a1. 1974: 361

Alessio et a1. 1974: 360

Alessio et al. 1974: 361

Alessio et al. 1974: 361

Alessio et a1. 1974: 361

Shotton et a1. 1970: 397

Barfield and Bagolini 1976: 140

Shotton et al. 1970: 397

Barfield and Bagolini 1976: 140

Alessio et a1. 1978: 81

Alessio et al. 1978: 81

Alessio et a1. 1978: 81

Bagolini and Biagi 1986: 387

Ferrara et al. 1959: 106

Ferrara et a1. 1959: 107

Guerreschi 1976-77: 494

Donati 1986: 109 
Site name

Monte Covolo

Völseraicha

DAN ILO CULTURE

Mitreo

Mitreo

CHASSEY AND LAGOZZA CULTURE
Uncal BP years

$4750 \pm 210$

$4750 \pm 100$

$5770 \pm 60$

$5770 \pm 50$

$5075 \pm 45$

$4995 \pm 100$

$4980 \pm 30$

$4980 \pm 50$

$4805 \pm 50$

$4794 \pm 90$

$4735 \pm 50$

$4580 \pm 50$

"CHALCOLITHIC"

Libiola

Libiola

Manerba

Laas

Va1 Frascarese

Val Frascarese

Monte Covolo

Spilamberto

Tolerait

Balm' Chanto

Balm' Chanto

Riparo Gaban

Tana de1 Barletta

BELL BEAKER CULTURE

Monte Covolo

Monte Covolo

Monte Covolo

Rubiera

S. Ilario

S. Ilario

S. Ilario

Ca' dei Grii
$4610 \pm 50$

$4490 \pm 90$

$4420 \pm 90$

$4350 \pm 100$

$4340 \pm 60$

$3920 \pm 60$

$4240 \pm 190$

$4195 \pm 95$

$4090 \pm 70$

$4010 \pm 60$

$3985 \pm 50$

$3880 \pm 120$

$4010 \pm 40$

$3950 \pm 320$

$3810 \pm 210$

$3900 \pm 60$

$3860 \pm 100$

$3840 \pm 100$

$3840 \pm 60$

$3900 \pm 80$
$4130 \pm 100$
Cal BC years

(1\%)

3760-3300

3680-3400

4737-4550

4729-4574

3968-3805

3950-3690

$3802-3722$

3807-3708

3807-3708

3687-3502

3626-3388

3372-3206

3382-3342

3354-3035

3309-2930

3090-2900

$3070-2590$

2917-2629

2900-2570

2871-2532

2603-2475

2581-2469

2520-2190

2593-2486

2920-2010

2550-1970

2481-2314

2490-2180

2480-2160

2455-2207
R-904

R-904A

Lab n

Birm-473

B1n-2715

R-104

I-11817

B-4562

$\mathrm{R}-338$

R-337

Pi-34

$\mathrm{R}-78$

R-78A

B1n-3367

Gif -7213

Birm-1132

Vri-272

B1n-3386

Gif-7212

Birm-472

I-11816

Birm-1005

B1n-3285

B 1 n- 2838

B1n-1776

Har -6435

GrN-8013

Birm-471

Birm-470

GrN-9828

Birm-828

Birm-827

R-1291

B1n-3753 
Material

Charcoa 1

charcoal

Charcoal

Charcoal

Charcoal

Charcoal

Charcoal

Wood

Wood

Wood

Wood

Wood

\section{Wood}

Wood

Wood

Charcoa1

Human bones

Human bones

Charcoal

Charcoal

Charcoal

Charcoal

Charcoal

Charcoal

Charcoa1

\section{Charcoa1}

Charcoal

Charcoal

Charcoal

Charcoal

Charcoa1

Charcoal

Human bones
Site type

Rock-she1ter

Open site

Cave
Cave

Cave

Open site

Open site

Lake dwe11ing

Lake dwelling

Lake dwelling

Lake dwe 11 ing

Lake dwe11ing

Layer 13-12

Site VIII

Layer 5

Post

Too 1

Post

Post

Post

Too 1

Too 1

Mine

Cemetery

open site

Cave

Cave

Open site

Open site

Open site

Rock-shelter

Rock-shelter

Rock-she 1 ter

Cave
Reference

Barfield et a1. 1977-79: 74

Bagolini and Biagi 1986: 387

Alessio et a1. 1978: 87

Alessio et a1. 1978: 87

Alessio et a1. 1966: 402

Bagolini 1981: 101

Donati 1986: 101

Alessio et a1. 1968: 356

Alessio et a1. 1968: 357

Ferrara et a1. 1961: 102

Alessio et a1. 1968: 356

Alessio et a1. 1968: 356

Maggi and Vigno1o 1989 (in press) Maggi and Vignolo 1989 in press: Barfield 1983: 122

Felber 1974: 227

Maggi 1989 (in press)

Maggi 1989 (in press)

Barfield et a1. 1977-79: 74

Bagolini 1989: 133

Biagi and Nisbet 1987: 16

Nisbet and Biagi 1987: 79

Nisbet and Biagi 1987: 78

Nisbet and Biagl 19.97: 78

Nishet and Biagi. 1987: 78

Layer 8

Layer 8

Layer 6

Barfield et a1. 1977-79: 74

Barfield et al. 1977-79: 74

Barfield et al. 1977-79: 74

Bermond Montanari et al. 1982: $10 \%$

Alessio et a1. 1980: 1423

Alessio et al. 1980: 1423

Alessio et a1. 1980: 1421

Biagi 1989 (in press) 
Figure 7

Distribution maps of the North Italian sites from which C14 dates were obtained. a) Late Mesolithic, b) Early Neolithic, c) Middle Neolithic, d) Late Neolithic, e) Chalcolithic, f) Bell Beaker Culture
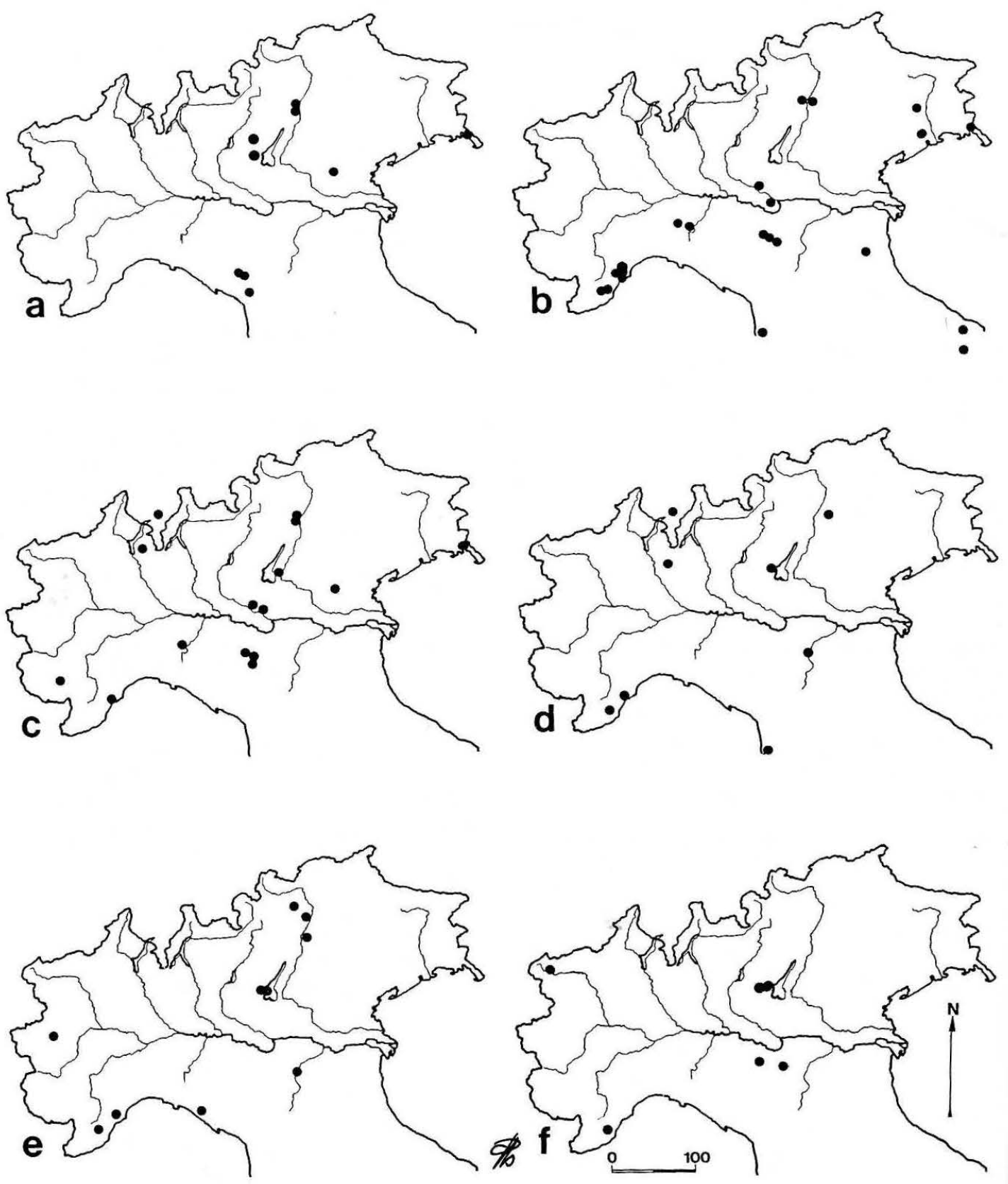
also decorate the stele-statues of Lunigiana in eastern Liguria and are well known in the alpine megaliths of Sion, Dolmen MVI (Bocksberger 1976) and in the rock engravings of Valcamonica and Valtellina (Anati 1974). Early fourth millennium BP dates come from the Beaker sites of Monte Covolo, Rubiera and Sant' Ilario (Barfield 1987) as well as from the burial cave Cà dei Grii (Biagi 1989), while those from the Beaker levels of Arma di Nasino in Liguria and the megalithic complex of Aosta are not considered acceptable. Also during the Chalcolithic, the Trieste Karst was half way between the north Italian and Dalmatian and Danubian world. In many caves the Copper Age is marked by Vučedol decorated pottery and by Besenstrich and Baden type sherds (Leben 1967). Even though no $\mathrm{C} 14$ date is yet available for the Karst Chalcolithic, we know that the Vučedol Culture in Bosnia belongs to the end of the fifth millennium BP, as reported by Quitta and Kohl (1969: 234), Bln-564: $4125 \pm 80$ BP, 2890-2593 BC (Fig. 7).

The appearance of the Bronze Age in northern Italy is characterised by the Polada and its related cultures, whose radiocarbon dates are generally more recent than the Beaker ones (Barfield 1974). ${ }^{1}$

\section{Acknowledgements}

The authors are very grateful to Drs $\mathbf{H}$. Quitta and K. Rassmann of the Academy of Sciences of GDR in Berlin for analysing the samples from many North Italian sites mentioned in the text.

Dipartimento di Storia della Cultura Europea Università di Trento (Bagolini) Dipartimento di Scienze Storico Archeologiche e Orientalistiche Università di Venezia (Biagi)

\section{NOTE}

1. All the dates mentioned in this paper are in uncalibrated BP years and in calibrated BC $(1 \sigma)$ years. Their presentation follows the Radiocarbon 1986 instructions (Stuiver and Reimer 1985). Apart from those mentioned in the text, a number of $\mathrm{C} 14$ dates are considered unacceptable to the present authors. They are: Fioranc (Bln-3137: 5570 $\pm 50 \mathrm{BP}$, Quitta pers. comm. 1985), Vhò Campo Sera Mattina (Birm-1234: $5570 \pm 200$ BP, Williams pers. comm. 1977), Arma dell'Aquila (Bln-3449: 4030 \pm 70 BP, Quitta pers. comm. 1986), Travo (Bernabò Brea et al. 1984: 76), La Vela (Shotton et al. 1970: 396), Rivaltella (Bagolini and Biagi 1986: 386), Fimon Molino Casarotto (Shotton et al. 1970: 397), Pertusello (Alessio et al. 1967: 348) and Civate (Alessio et al. 1976: 324).

This paper is published with the aid of a $60 \%$ MPI grant. The chapters on the Mesolithic and Neolithic are by $P$. Biagi, that on the Chalcolithic is by B. Bagolini.

\section{REFERENCES}

ACCORSI, C.A., BANDINI MAZZANTI, C., BIAGI, P.,
CASTELLETTI, L., CREMASCHI, M., LEONI, L. and PAVARANI, M. 1976: Il sito mesolitico sopra Fienile Rossino sull'Altipiano di Cariadeghe (Serle-Brescia). Aspetti pedostratigrafici, archeologici, antracologici e palinologici. Natura Bresciana 23, 239-292.

ALESSIO, M., ALlegri, L., Bella, F., BROGLIO, A., CALDERONI, G., CORTESI, C., IMPROTA, S., PREITE MARTINEZ, M., PETRONE, v. and TURI, B. 1983: 14C datings of three mesolithic series of Trento basin in the Adige Valley (Vatte di Zambana, Pradestel, Romagnano) and comparisons with mesolithic series of other regions. Preistoria Alpina 19, 245-254.

Alessio, M., Allegri, L., Bella, E., CALDERONI, G., CORTESI, C., CREMASCHI, M., IMPROTA, S., PAPANI, G. and PETRONE, V. 1980: Le datazioni 14C della pianura tardowurmiana ed olocenica nell'Emilia Occidentale. In Contributi alla realizzazione della carta neotettonica d'Italia, Parte II. CNR, Progetto Finalizzato Geodinamica. Sottoprogetto 'Neotettonica' 356, 1411-1435.

ALESSIO, M., ALlEGRI, L., BELLA, F., IMPROTA, S., BELLUOMINI, G., CALDERONI, G., CORTESI, C., MANFRA, L. and TURI, B. 1978: University of Rome Carbon-14 Dates XVI. Radiocarbon 20(1), 79-104. 
ALESSIO, M., BELLA, F., BACHECHI, F. and CORTESI, C. 1965: University of Rome Carbon-14 Dates III. Radiocarbon 7, 213-222.

ALESSIO, M., BELLA, F., BACHECHI, F. and CORTESI, C. 1966: University of Rome Carbon-14 Dates IV. Radiocarbon $8,401-412$.

ALESSIO, M., BELLA, F., CORTESI, C. and GRAZIADEI, B. 1968: University of Rome Carbon-14 Dates VI. Radiocarbon 10(2), 350-364.

ALESSIO, M., BELlA, F., IMPROTA, S., BELLUOMINI, G., CORTESI, C. and TURI, B. 1970: University of Rome Carbon-14 Dates VIII. Radiocarbon 12, 599-616.

ALESSIO, M., BELlA, F., IMPROTA, S., BELLUOMINI, G., CORTESI, C. and TURI, B. 1974: University of Rome Carbon-14 Dates XII. Radiocarbon 16(3), 358-367.

ALLEGRI, L., CORTESI, C. and RADMILLI, A.M. 1987: La cronologia neolitica in base al radiocarbonio. Atti XXVI Riunione Scientifica IIPP, 67-77.

ANATI, E. 1974: Le figurazioni di pugnali della Valcamonica. Preistoria Alpina 10, 133-136.

ANTONIAZZI, A., BAGOLINI, B., BERMOND MONTANARI, G., MASSI PASI, M. and PRATI, L. 1986: Die Jungsteinzeit in Fornace dei Cappuccini in Faenza und die Impressokeramik in der Romagna. Jahrbuch des Béri-BaloghÁdám Museums 13, 337-363.

BAFFICO, O., BIAGI, P. and MAGGI, R. 1983: Il Mesolitico. In R. Maggi (ed.) Preistoria nella Liguria Orientale, 33-44 (Chiavari, Siri).

BAGOLINI, B. 1981: Il sepolcreto e gli insediamenti eneolitici di Spilamberto S. Cesario nel quadro culturale mediopadano. In B. Bagolini (ed.) Il Neolitico e l'Età del Rame. Ricerca a Spilamberto e S. Cesario 19771980, 217-276 (Bologna, Tamari).

BAGOLINI, B. 1986: Westliche Einflusse im Neolithikum in Norditalien. Jahrbuch des Béri-Balogh-Ádám Museums $13,365-371$.

BAGOLINI, B. 1989: La necropoli neolitica de La Vela di Trento. In P. Biagi (ed.) International Round Table 'The Neolithisation of the Alpine Region', in press (Brescia).

BAGOLINI, B., BARBACOVI, F. and BIAGI, P. 1979: Le Basse di Valcalaona (Colli Euganei). Alcune osservazioni su una facies con vasi a bocca quadrata e sulla sua collocazione cronologico culturale. Monografie di Natura Bresciana 3.

BAGOLINI, B., BARFIELD, L.H. and BROGLIO, A. 1973: Notizie preliminari delle ricerche sull'insediamento neolitico di Fimon Molino Casarotto (1969-1972). Rivista di Scienze Preistoriche 27, 161-215.

BAGOLINI, B. and BIAGI, P. 1976: La Vela de Trente et le 'moment de style Adriatique' dans la Culture des Vases a Bouche Carrée. Preistoria Alpina 12, 71-77.

BAGOLINI, B. and BIAGI, P. 1986: Chronology and distribution of the Square Mouth Pottery Culture settlements of Northern Italy. Jahrbuch des Béri-BaloghÁdám Museums 13, 373-387.

BAGOLINI, B. and BIAGI, P. 1987a: The first Neolithic Chipped Stone Assemblages of Northern Italy. In Kozlowski, J.K. and Kozlowski, S.K. (eds.) Chipped Stone Assemblages of the Early Farming Cultures in Europe, 423-448 (Warsaw).

BAGOLINI, B. and BIAGI, P. 1987b: Il Neolitico dell'EmiliaRomagna. Atti XXVI Riunione Scientifica IIPP, 217-227.

BAGOLINI, B., BRESSAN, F. and CANDUSSIO, A. 1987: Prima campagna di scavi a Sammardenchia di Pozzuolo del Friuli (Udine). Atti XXVI Riunione Scientifica IIPP, 407-412.

BAGOLINI, B., BROGLIO, A. and LUNZ, R. 1983: Le Mésolithique des Dolomites. Preistoria Alpina 19, 15-136.

BAGOLINI, B. and VON ELES, P. 198: L'insediamento neolitico di Imola e la corrente culturale della Ceramica Impressa nel medio e alto Adriatico. Preistoria Alpina $14,33-63$.

BARFIELD, L.H. 1969: Two italian halberds and the question of the earliest European halberds. Origini 3, 67-83.

BARFIELD, L.H. 1970: La stazione neolitica de 'La Vela' presso Trento. Considerazioni sulle tombe a cista nel Trentino. Studi Trentini di Scienze Naturali B, 47(1), 35-55.

BARFIELD, L.H. 1973: The Square-Mouthed Pottery Culture in the Veneto and its Adriatic Connections. In Actés VIII Congrés UISPP 2, 393-398 (Beograd).

BARFIELD, L.H. 1974: The cultural affinities of Bell Beakers in Italy and Sicily. In J.N. Lanting and J.D. Van der Waals (eds.) Glockenbechersimposion, 307-322 (Oberried).

BARFIELD, L.H. 1981: Patterns of North Italian Trade 5000-2000 bc. In G. Barker and R. Hodges (eds.) Archaeology and Italian Society. Papers in Italian Archaeology. BAR International Series 102, 27-51.

BARFIELD, L.H. 1983: The Chalcolithic Cemetery at 
Manerba del Garda. Antiquity 57, 116-123.

BARFIELD, L.H. 1985: Burials and Boundaries in Chalcolithic Italy. In C. Malone and S. Stoddard (eds.) Papers in Italian Archaeology IV, Part II Prehistory. BAR International Series 244, 152-176.

BARFIELD, L.H. 1987: The Italian dimension of the Beaker problem. In W.H. Waldren and R.C. Kennard (eds.) Bell Beakers of the Western Mediterranean. BAR International Series 331, 499-515.

BARFIELD, L.H. and BAGOLINI, B. 1976: The excavations on the Rocca di Rivoli, Verona 1963-1968. Memorie del Museo Civico di Storia Naturale di Verona, Sezione Scienze dell'Uomo 1.

BARFIELD, L.H., BARKER, G.W.W., CHESTERMAN, J.T., PALS, J.P. and VOORRIPS, A. 1977-79: Excavations at Monte Covolo, Villanuova sul Clisi, Brescia (1972-73), Part II. Annali del Museo Gavardo 13, 5-89.

BARFIELD, L.H. and BROGLIO, A. (eds.) 1986: L'insediamento neolitico di Molino Casarotto nelle Valli di Fimon (Colli Berici, Vicenza). Parte I (Ferrara, Tosi).

BARKER, G.W.W. 1975: Prehistoric territories and economies in Central Italy. In E.S. Higgs (ed.) Palaeoeconomy, 111-176 (Cambridge, University Press).

BARONI, C., BIAGI, P., NISBET, R. and SCAIFE, R.G. 1989: Laghetti del Crestoso: a high altitude Castelnovian camp on its environment setting. In P. Biagi (ed.) International Round Table 'The Neolithisation of the Alpine Region', in press (Brescia).

BERMOND MONTANARI, G., CREMASCHI, M. and SALA, B. 1982: Rubiera: insediamento del vaso campaniforme. Preistoria Alpina 18, 79-109.

BERNABO' BREA, L. 1956: Gli scavi nella Caverna delle Arene Candide. Parte I. Gli strati con ceramiche 2 (Bordighera, Istituto di Studi Liguri).

BERNABO' BREA, M. 1986: Ricerche sugli insediamenti neolitici della media Val Trebbia. Dialoghi di Archeologia $4(2), 123-177$.

BERNABO' BREA, M., CATTANi, M., CONVERSi, R., CREMASCHI, M., NISBET, R. and RICCI, C. 1984: L'insediamento neolitico della Cassa di Risparmio a Travo (PC). Preistoria Alpina 20, 59-80.

BIAGI, P. 1979: Stazione neolitica a Ostiano (CR), località Dugali Alti: 1980. Preistoria Alpina 15, 28-38.

BIAGI, P. 1980: Some aspects of the Prehistory of Northern Italy from the Final Palaeolithic to the Middle
Neolithic: A Reconsideration on the Evidence Available to Date. Proceedings of the Prehistoric Society 46, 9-18.

BIAGI, P. 1982: Observations on the Late Neolithic of Northern Italy. Symposia Thracica A', 77-82.

BIAGI, P. 1985: Neue Aspekte zur Neolithisierung Norditaliens. Zeitschrift für Archäologie 19(1), 11-22.

BIAGI, P. 1987a: Il Neolitico della Liguria e del Piemonte. Atti XXVI Riunione Scientifica IIPP, 203-215.

BIAGI, P. 1987b: Aspetti dell'Archeologia in Lombardia: IX-IV millennio bc. Atti II Covegno Archeologico Regionale, 379-396 (Como, New Press).

BIAGI, P. 1989: Una datazione radiocarbonica dalla Cavernetta Ca' dei Grii (n. 66 Lo) (Virle, Brescia). Natura Bresciana 25 (in press).

BIAGI, P., CASTELletTI, L., CREMASCHI, M., SAlA, B. and TOZZI, C. 1980: Popolazione e territorio nell'Appennino Tosco-Emiliano e nel tratto centrale del bacino del Po tra il IX ed il V millennio. Emilia Preromana 8, 13-36.

BIAGI, P., MAGGI, R. and NISBET, R. 1987: Excavations at Arma dello Stefanin (Val Pennavaira - Albenga, Northern Italy) 1982-86. Mesolithic Miscellany 10(1), $10-11$.

BIAGI, P., MAGGI, R. and NISBET, R. 1989: Liguria: 11,000-7000 BP. In C. Bonsall (ed.) The Mesolithic in Europe. III International Symposium (Edinburgh), 533-40.

BIAGI, P. and NISBET, R. 1986: Popolazione e territorio in Liguria tra il XII e il IV millennio bc. In Scritti in ricordo di G. Massari Taballo e U. Tocchetti Pollini, 19-27 (Milano, ET).

BIAGI, P. and NISBET, R. 1987: Ursprung der Landwirtschaft in Norditalien. Zeitschrift für Archäologie 21(1), 11-24.

Bocksberger, O-J. 1976: Le site Préhistorique du PetitChasseur (Sion, Valais), 2. Le Dolmen MVI. Documents du Département d'Anthropologie de l'Université de Genève 2 (Lausanne).

BROGLIO, A. 1971: Risultati preliminari delle ricerche sui complessi epipaleolitici della Valle dell'Adige. Preistoria Alpina 7, 135-241.

BRoglio, A. 1972: Cronologia delle Culture del Paleolitico superiore, dell'Epipaleolitico e del Neolitico della Val Padana. Bollettino Centro Camuno Studi Preistorici 8, 47-79.

BROGLIO, A. and LOLLINI, D.G. 1963: Nuova varietà di 
Bulino su ritocco a stacco laterale nell'industria del Neolitico medio di Ripabiancà di Monterado (Ancona). Annali Università di Ferrara, Sezione 15, 1, 143-155.

CANNARELLA, D. and CREMONESI, G. 1967: Gli scavi nella Grotta Azzurra di Samatorza nel Carso Triestino. Rivista di Scienze Preistoriche 22(2), 281-330.

CATTANI, L. 1977: La Grottina dei Covoloni del Broion (Colli Berici, Vicenza). Analisi pollinica. Rivista di Scienze Preistoriche 32, 287-296.

COCCHI GENICK, D. and GRIFONI CREMONESI, R. (eds.) 1985: L'eià dei metalli nella Toscana nord-occidentale (Pisa, Pacini).

COLINI, G. 1898-1902: Il sepolcreto di Remedello Sotto nel Bresciano ed il periodo eneolitico in Italia. Bullettino di Paletnologia Italiana 24, 1-110; 25, 1-32, 218-311; 26, 57-101, 202-267; 27, 73-132; 28, 5-43, 66-103.

CREMASCHI, M., BIAGI, P., ACCORSI, C.A., BANDINI MAZZANTI, M., RODOLFI, G., CASTELLETTI, L. and LEONI, L. 1981-82: Il sito mesolitico di Monte Bagioletto (Appennino Reggiano) nel quadro delle variazioni ambientali oloceniche dell'Appennino Tosco Emiliano. Emilia Preromana 9/10, 11-46.

CREMONESI, G. 1978-81: Caratteristiche economico industriali del Mesolitico del Carso. Atti Società di Preistoria e Protostoria del Friuli Venezia Giulia 4, 171-186.

DONATI, P. 1986: Bellinzona a Castel Grande. 6000 anni di storia. Archaeologie der Schweiz 9(3), 94-109.

FELBER, H. 1974: Vienna radiocarbon Institute, Radiocarbon Dates V. Radiocarbon 16, 277-283.

FERRARA, G., FORNACA RINALDI, G. and TONGIORGI, E. 1961: Carbon 14 Dating in Pisa II. Radiocarbon 3, 99-103.

FERRARA, G., REINHARZ, M. and TONGIORGI, E. 1959: Carbon 14 Dating in Pisa. Radiocarbon 1, 103-110.

GEDDES, D.S., GUILAINE, J. and MONACO, A. 1983: Early Neolithic occupation on the submerged continental plateau of Roussillon (France). In P.M. Masters and N.C. Fleming (eds.) Quaternary Coastlines and Marine Archaeology. Towards the Prehistory of Land Bridges and Continental Shelves, 175-187 (London, Academic Press).

GIROD, A. 1989: La malacofauna olocenica dell'Arma dell'Aquila (Finale Ligure, Savona). Natura Bresciana 25 (in press).

GUERRESCHI, G. 1976-77: La stratigrafia dell'Isolino di
Varese dedotta dall'analisi della ceramica (scavi Bertolone 1955-1959). Sibrium 13, 29-528.

ISETTI, G. 1964: Il rame dei Tigullii e il problema di Chiavari. Rivista Studi Liguri 30 (1/4), 83-90.

ISSEL, A. 1892: Liguria Geologica e Preistorica (Genova).

LEBEN, F. 1967: Stratigrafija in časovna uvrstitev jamskih najdb na Tržaškem Krasu. Arheološki Vestnik 18, 43-109.

LEWTHWAITE, J. 1981: Ambiguous first impressions: a survey of recent work on the Early Neolithic of the West Mediterranean. Journal Mediterranean Anthropology Archaeology 1(2), 292-307.

LEWTHWAITE, J. 1985: From precocity to involution: the Neolithic of Corsica in its Mediterranean and French contexts. Oxford Journal of Archaeology 4(1), 47-68.

LEWTHWAITE, J. 1986: The transition to food production: A Mediterranean perspective. In M. Zvelebil (ed.) Hunters in Transition. Mesolithic Societies of Temperate Europe and their Transition to Farming, 53-66 (Cambridge, University Press).

MAGGI, R. 1977: Lo strato a ceramiche graffite delle Arene Candide. Preistoria Alpina 13, 205-211.

MAGGI, R. 1989: Val Frascarese. Caverna sepolcrale 'Da Prima Ciappa'. Datazioni radiocarboniche su ossa umane. Archeologia in Liguria 3(1) (in press).

MAGGI, R. and FORMICOLA, v. 1978: Una grotticella sepolcrale dell'età del Bronzo in Val Frascarese (Genova). Preistoria Alpina 14, 87-113.

MAGGI, R. and VIGNOLO, M.R. 1989: Libiola (Sestri Levante - GE). Archeologia in Liguria 3(1) (in press).

mezzena, F. 1981: La Valle d'Aosta nella Preistoria e nella Protostoria. Archeologia in Valle d'Aosta, 15-60 (Aosta).

MILLS, N. 1983: The Neolithic of Southern France. In Scarre, C. (ed.) Ancient France 6000-3000 BC, 91-145 (Edinburgh, University Press).

NIEDERWANGER, G. 1984: Ur- und Frühgeschichte des Sarntals. Archaeologische-Historische Forschungen in Tirol 8 (Trento, Manfrini).

NISBET, R. and BIAGI, P. (eds.) 1987: Balm' Chanto: un riparo sotto roccia dell'età del Rame nelle Alpi Cozie. Archeologia dell'Italia Settentrionale 4 (Como, New Press).

ODETTI, G. 1984: Ricerche preistoriche in Val Maremola: 
la Grotta del'Ėdera. Rivista Ingauna e Intemelia 39(3/4), 102-116.

ORME, B. 1981: Anthropology for Archaelogists (London, Duckworth).

OTTAWAY, B. 1973: Dispersion diagrams: a new approach to the display of Carbon-14 dates. Archaeometry 15(1), 5-12.

QUITTA, H. and KOHL, G. 1969: Neue Radiocarbondaten zum Neolithikum und zur frühen Bronzezeit Südosteuropas und der Sowjetunion. Zeitschrift für Archäologie 3, 223-255.

RADMILLI, A.M. 1963: Il Mesolitico nel Carso Triestino. Atti VII Riunione Scientifica IIPP, 39-43.

RENFREW, C. and WhITEHOUSE, R. 1974: The Copper Age of Peninsular Italy and the Egean. Annals British School at Athens 69, 343-390.

SARGENT, A. 1985: The Carbon-14 Chronology of the Early and Middle Neolithic of Southern Italy. Proceedings of the Prehistoric Society 51, 31-40.

SHOTTON, R.W., BLUNDELL, D.J. and WILLIAMS, R.E.G. 1970: Birmingham University Radiocarbon Dates IV. Radiocarbon 12(2), 385-399.
SHOTTON, F.W. and WILLIAMS, R.E.G. 1973: Birmingham University Radiocarbon Dates VI. Radiocarbon 15(1), $1-12$.

SIMONE, L. 1987: Une cabanne du Néolithique Ancien dans l'Oltrepò Pavese (Lombardie). In J. Guilaine, J. Combier, J-L. Roudil and J-L. Vernet (eds.) Premières Communautés Paysannes en Mediterranée Occidentale, 471-474 (CNRS, Paris).

STUIVER, M. and REIMER, P.J. 1986: A computer program for radiocarbon age calibration. Radiocarbon 28(2B), 1022-1030.

TINE', S. 1974: Il Neolitico e l'età del Bronzo in Liguria alla luce delle recenti scoperte. Atti XVI Riunione Scientifica IIPP, 37-57.

Whitehouse, R. 1978: Italian Prehistory, Carbon-14 and the Tree-ring Calibration. In H.McK. Blake, T.W. Potter and D.B. Whitehouse (eds.) Papers in Italian Archaeology I: The Lancaster Seminar. BAR International Series 41, 71-91.

WHITEHOUSE, R. 1985: Nuove date al C14 del Neolitico nell'Italia Orientale. Lancaster in Italy 1984, 35-39. 